\title{
Body Contouring: Combined Lower Circumferential Torsoplasty and Inferior Pedicle Mastopexy in Males: Male Lift-up Surgery
}

\author{
Abdulaziz Alrasheed ${ }^{1}$ and Rami Makkar ${ }^{2 *}$ \\ ${ }^{1}$ Head, Department of Plastic Surgery, Kuwait National Guard, Kuwait \\ ${ }^{2}$ Associate Professor, Department of Plastic Surgery, School of Medicine, Cairo University, Egypt
}

\begin{abstract}
Background: Male population experienced body contour deformities secondary to massive weight loss. Body-contouring surgery for chest and abdominal wall deformities in massive weight loss patient need wise preoperative evaluation and planning.
\end{abstract}

Methods: For correction of these common deformities, combined bilateral mastopexy with inferior based pedicle and circumferential abdominoplasty single stage surgery safety and outcome for 60 male patients were evaluated.

Results: This combination successfully improved the contour of the anterior and lateral chest as well as the anterior abdomen and back in one go.

Conclusion: The procedure showed no significant increase in post-operative complications and is safe to achieve satisfactory result.

Keywords

Gynecomastia, Male Contouring, Upper Torsoplasty

\section{Introduction}

Male patients following massive weigh loss through diet and exercising suffer from excess of skin, primarily at the abdomen and breast region. Those patients are contented with the progress in losing weight; however they are primarily bothered by the skin laxity and excess of fat at the abdominal wall and breast region and become frustrated by the developing fatty apron and the breast's skin behave identically like in females after weight loss, resulting in breast ptosis with caudal displacement of the IMF [1].

Psychological distress caused by acquiring a pendulous female breast shape and Physical impairments such as sore skin, difficulties with exercise, or problems to find suitable clothes are reasons for the strong desire of patients for surgery [2]. The increased media coverage of aesthetic plastic surgery and the popularization of the "makeover" concept, the immediate gratification, expedited recovery, and financial savings associated with these procedures have become powerful motivators for patients to request combined cosmetic surgery.

Abdominoplasty and cosmetic breast surgery (breasts lifts) are the most common plastic surgical procedures after massive weight loss when performed in one operation, exem- plifies this concept $[2,3]$.

The safety of combined aesthetic procedures has been addressed in the plastic surgery literature with no significant differences between single and combined procedure with regard to either minor or major complications [4].

The goal of this study is to present our experience and technique to reach a good aesthetic result in one go with the minimal possible complication rate to improve patients' satisfaction and reduce the number of anesthesia.

\section{Material and Methods}

The procedure was performed on 60 male patients presenting with pseudogynecomastia with NAC and IMF below

*Corresponding author: Rami Makkar, Associate Professor, Department of Plastic Surgery, School of Medicine, Cairo University, Egypt, Tel: 01222318992, Fax: 60015613

Accepted: October 06, 2020

Published online: October 08, 2020

Citation: Alrasheed A, Makkar R (2020) Body Contouring: Combined Lower Circumferential Torsoplasty and Inferior Pedicle Mastopexy in Males: Male Lift-up Surgery. J Cosmet Surg 1(1):5-4 

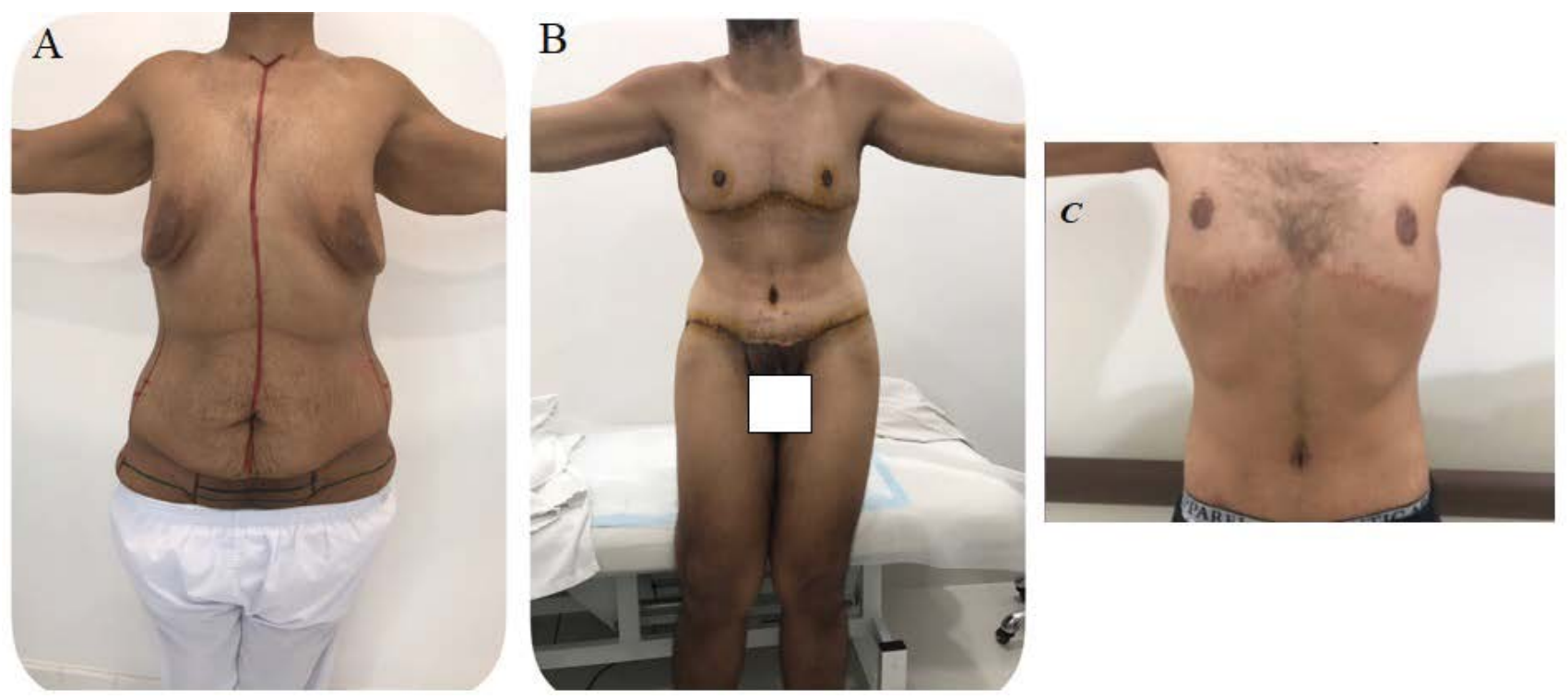

Figure 1: Examination and Marking. Are best applied while the patient is standing and bending forward to reveal the extent of sagging skin.

the ideal IMF, lateral chest roll, and minimal and significant upper abdominal laxity (Grade 2 and 3) [5]. Those patients have significant truncal skin redundancy mainly over the abdomen and back following massive weight loss. The procedure was done in private hospitals in Kuwait by both authors' team from January-2014 to January-2017. Preoperative routine medical evaluation and clearance were done for all patients.

Although the lower and upper body operations may be performed separately, they are best planned together, as each excision interrelates to achieve an overall tight skin envelope throughout the torso.

First, the markings for the $270^{\circ}$ extended abdominoplasty (circumferential) are drawn with the patient reclined and standing following the guideline of Al Rasheed, et al. as usual [6].

Second, the patient's IMF is indicated beneath the breasts on both sides. The invariably descended IMFs are positioned upwards. A simple pattern (incomplete horizontal ellipse) is applied to design the extent of the excision by pushing and grasping the tissues while the patient is standing and bending forward (Figure 1) to reveals the extent of the sagging skin. This ellipse is combined with the vertical ellipse centered on the mid-axillary line following the silhouette of the pectoralis major muscle reaching up to the axilla. With this sweeping extension toward the axilla, the excision resembles to a "Golf stick". The inferiorly based full thickness skin pedicle harboring the NAC is also marked. This vertical mid lateral chest excision tightens more the skin covering the chest giving a more masculine appearance. Strict safety guidelines [7] for all our patients were applied to minimize major and minor complications.

The patient was initially positioned prone for excision of the redundant skin of the back and flank. In the supine position before the traditional abdominoplasty is carried on, the extent of the previously marked excision of the abdomen and breast is revised together using towel clips or skin staplers to ensure the width of the ellipses which may be readjusted upon. Following the abdominoplasty, the breast skin excision is carried on. The vertical mid lateral chest excision is continued superficially as we advance toward the axilla. The nipple-areola complex is relocated into a receiving dome after excision of the remaining breast tissue and minimal defatening of the superior flap. The NAC is located $4 \mathrm{~cm}$ from the resulting scar. 2 closed system drains are applied. The patients were placed in bed, in full garments, with their hips flexed and were encouraged to mobilize for early ambulation as soon as possible.

\section{Results}

60 patients underwent combined lower circumferential Torsoplasty and inferior pedicle mastopexy. The preoperative data were collected from the medical records. Age, smoking history, weight loss before surgery and BMI at surgery, type of obesity-related surgery. Intraoperative blood loss, the need for blood transfusion and operative time were recorded. All patients were examined regularly post-operative until complete wound healing was achieved. Wound healing complications, late complications, and reversional surgeries were documented.

The preoperative patients' ages and BMI ranged from 23 to 54 years, and 25 to 32 years, respectively. The average operative time was 3.2 hours (range, 3 to 4 hours). The estimate blood loss ranged from 1.3 to 1.8 litters and $11 \%$ required blood transfusion either intraoperative or early post-operative. All patients were hospitalized and remained inpatients for an average of 4 days (range, 4 to 5 days). The average follow-up period was 18 months (range 7 months to 1.5 -years).

The normal sensation and pigmentation over the nipple areola complex was perfect in $98 \%$ of cases. Widening (enlarged) and ptotic NAC deformities resulted in 2 and 1 cases respectively that required revision by benelli mastopexy. The 
Citation: Alrasheed A, Makkar R (2020) Body Contouring: Combined Lower Circumferential Torsoplasty and Inferior Pedicle Mastopexy in Males: Male Lift-up Surgery. J Cosmet Surg 1(1):5-4

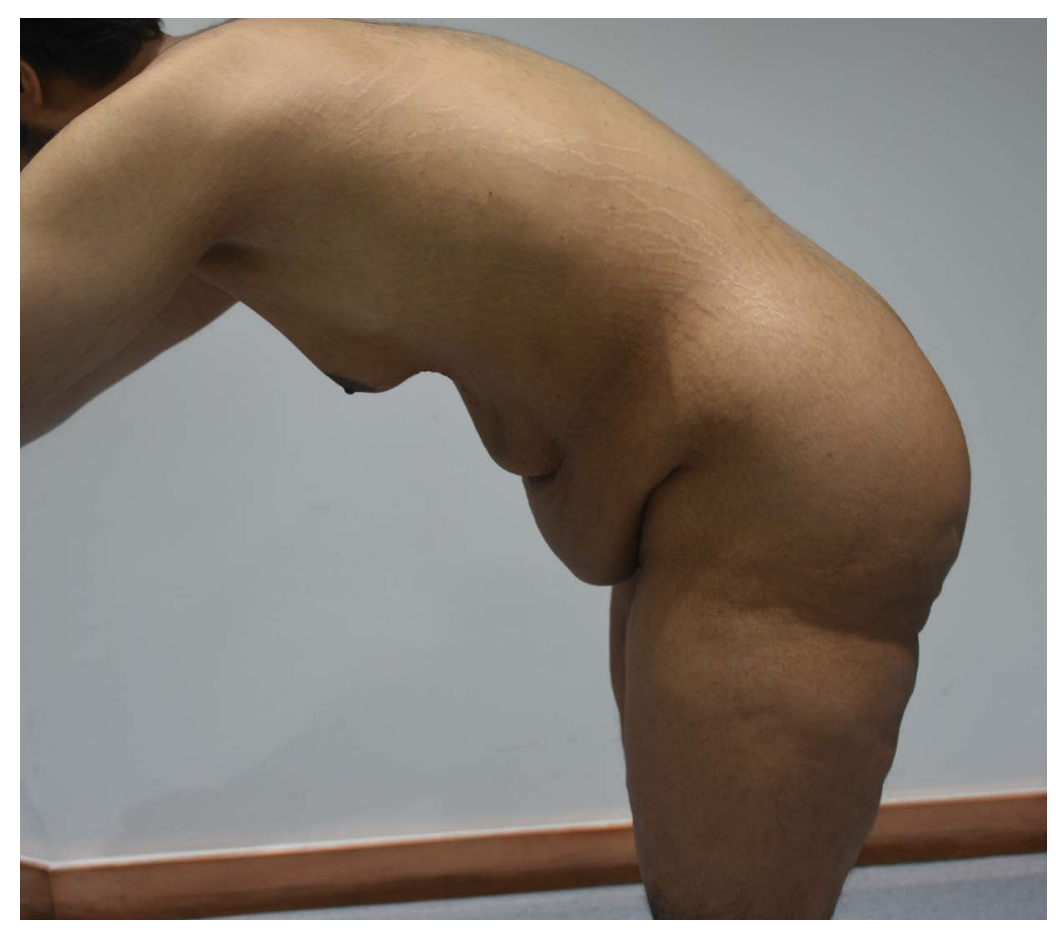

Figure 2: Case $1(A, B)$ Pre and 10 days Post-operative result of combined procedure; (C) 1-year Post-operative result.
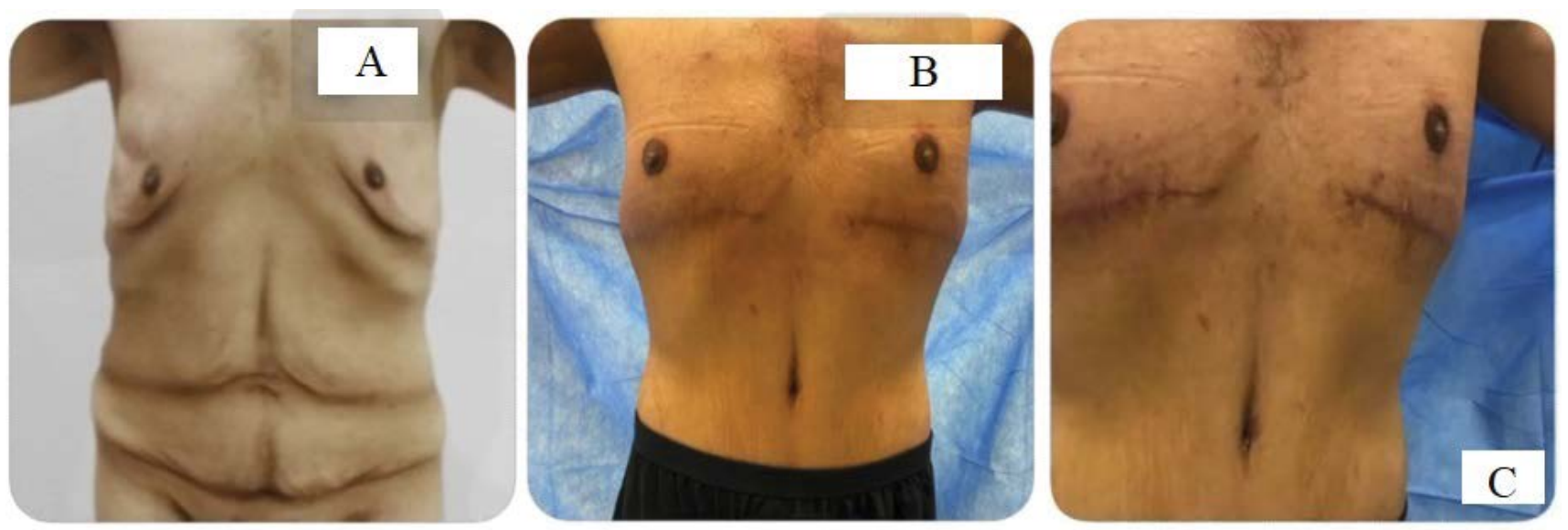

Figure 3: Case 2- Before (A) and After (B) 1-year post-surgery with a close view (C) of the scar.
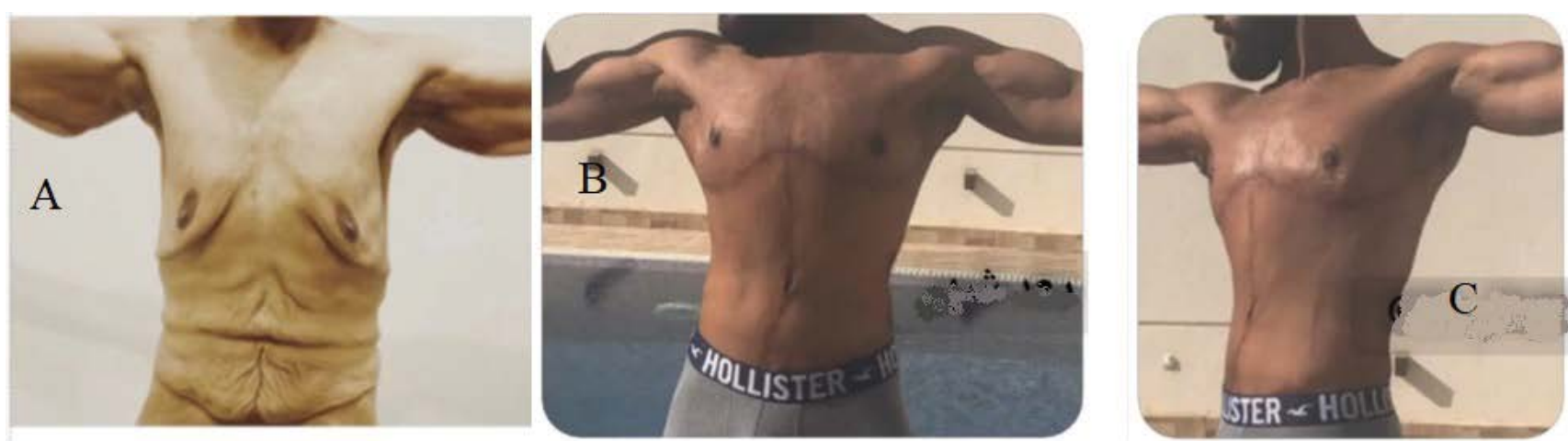

Figure 4: Case 3- Pre (A) and Post (B) operative 1-year result (C) the ideal male breast consists of trained and hypertrophic pectoralis major muscle and a NAC that is situated above the sub-mammary fold, which correspond to the inferior border of the pectoralis major muscle. 
scars were accepted except for hypertrophic scars (4 cases) that required revision too under local anesthesia. Overall revisional surgeries were $11 \%$. Seromas developed in 5 cases (8.3\%) and hematomas in 2 cases (3.3\%) and were treated conservatively with frequent aspiration and applying compression garments.

The complications of wound healing disorders (Clavien-Dindo classification grade - I) [8] in the form of necrotic skin edges, small wound dehiscence's, and minor skin slough were left to heal spontaneously with frequent dressings. Skin necroses larger than $2 \mathrm{~cm}$ were debrided, followed by secondary skin closure. Local infection was treated with broad-spectrum antibiotic followed by a specific antibiotic treatment as determined by the microbiologic findings. There were neither major complications nor deaths.

Overall, Patients were uniformly pleased with their results, the patients claimed they acquired the desired functional and aesthetic outcome. Their quality of life, and self-confi- dence has improved and regain their physical activity shortly post-operative in 6 weeks (Figure 2, Figure 3, Figure 4 and Figure 5).

\section{Discussion}

The number of men presenting for body contouring after massive weight grows [9]. Abdominoplasty and breast procedures together, account for the majority of procedures in worldwide plastic surgery [10].

A common deformity seen in the male population is an excess in redundant skin of the chest wall giving the look of female breast. This most often represents pseudogynecomastia. Pseudogynecomastia is characterized by increased subareolar fat without enlargement of the breast glandular component [5]. As in our series, massive loss of weight increases the skin redundancy with displacement of the IMF caudally and necessitates skin reduction and repositioning of the IMF to allow immediate breast recontouring.

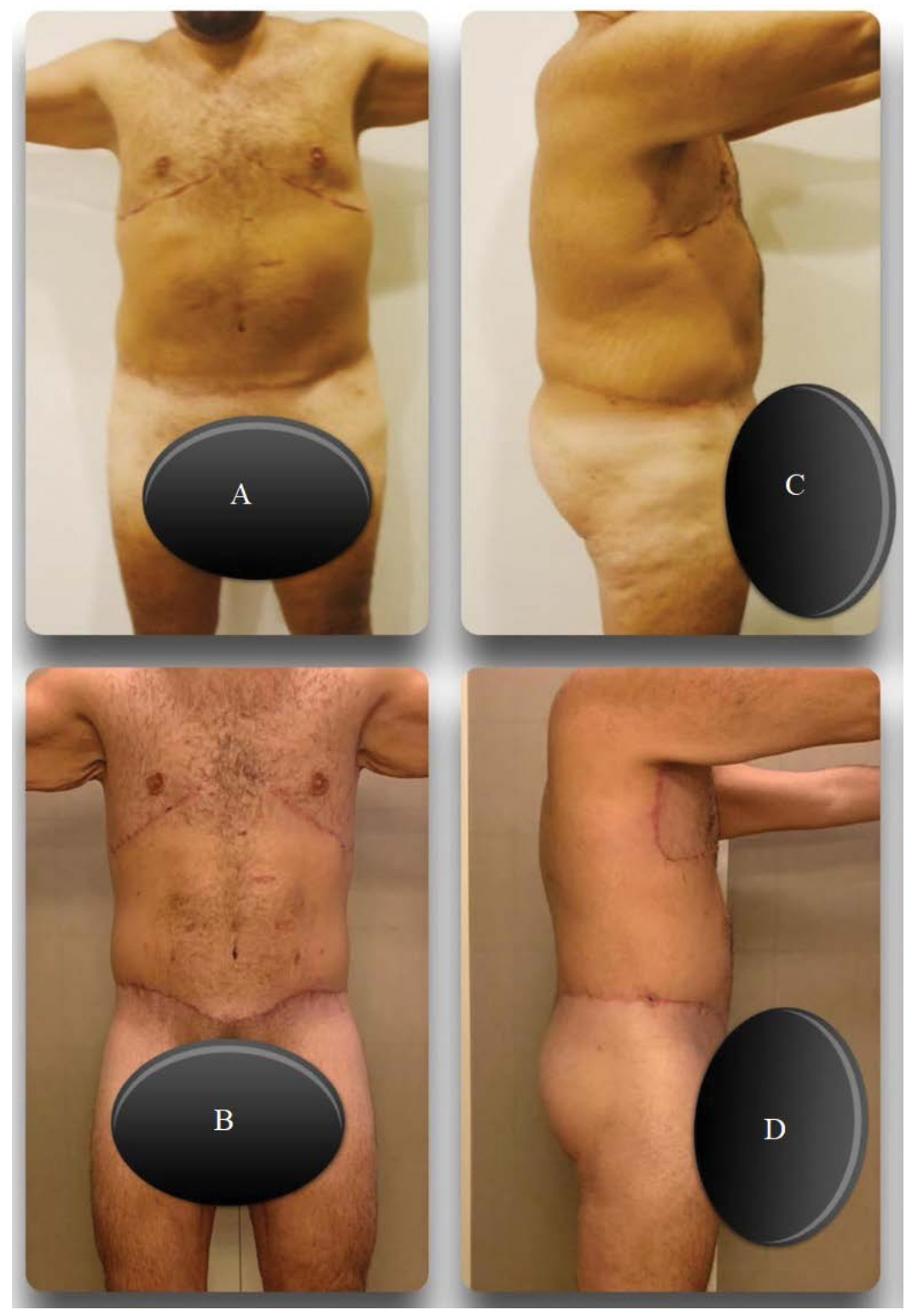

Figure 5: Case 4- Anterior and Lateral views for Pre and 3 Months Post-Operative. 
Citation: Alrasheed A, Makkar R (2020) Body Contouring: Combined Lower Circumferential Torsoplasty and Inferior Pedicle Mastopexy in Males: Male Lift-up Surgery. J Cosmet Surg 1(1):5-4

According to Grazer [11] the reverse abdominoplasty may be combined with a breast mastopexy for correction of upper abdominal skin laxity. It consisted of a hemi circumferential anterior incision along the IMF and elevation of an inferior flap of the epigastrium to the costal margins, followed by transverse excision of distal excess skin. In our series, we followed the same concept but the transverse excision was tailored to embrace the elliptical excision for the treatment of the male chest deformity taking into consideration the excess tissue of the chest in vertical and horizontal dimensions [12]. The scar follows the silhouette of the pectoralis muscle: It courses with this horizontal ellipse at the level of the sub mammary fold with an additional vertical scar on the lateral chest wall toward the axilla for enhancement of the breast lift. It is critical that the dissection be minimally traumatic, and the wound be securely closed under maximal tension.

The concept of complete correction of the deformities is preferred by almost all patients through one major operative intervention instead of two or more [12]. Combined-breast and abdominal procedure is not a new concept. Rao [13] described it for the first time in 1969. In men, a single-stage surgery has technical advantages. Failure to address the entire aesthetic unit of the chest in continuity with the lateral chest and abdomen can lead to suboptimal results [5].

Circumferential skin excess of the trunk and buttocks was corrected by circumferential resection according to the operative design. Gynecomastia with inelastic skin was corrected by bilateral mastopexy. The IMF is obliterated, and the chest wall and upper abdominal skin are red raped. Since male breasts have to be significantly reduced in volume and skin surface, ideally with preservation of the nipple-areola complex, the inferiorly based pedicle in a horizontal elliptical chest wall excision pattern, extending vertically toward the axilla was our technique to achieve the desired functional and aesthetic improvement. This technique with inferior pedicle can be performed in almost all cases.

It has been said because of excessive blood loss and prolonged operative and anesthesia time, such an approach may cause increased morbidity and delay convalescence. On the contrary, our result shows that not all patients received blood transfusion, all patients have a smooth post-operative convalescence and the average time for surgery and anesthesia were 3.5 hours which is the average time for a single cosmetic procedure if the surgery was well planned, done by a well-trained team of surgeons with precise and meticulous surgical technique. Interestingly, we noted that the wound healing complication rate was not highest in the longest operations as previously mentioned by Gmür in 2003 [14]. Wound healing complications, (Clavien-Dindo classification grade -1 ) late complications, and revisional surgery, as well as the length of hospital stay and the number of follow-up visits, were in the normal average. We also noted that the more weight the patient has lost before the operation, the better the outcome. We have achieved a curved sub mammary fold, starting slightly lateral to the midline, and descending to the breast median, smoothly ascending to the anterior axillary line. and a well-positioned NAC that is situated close above the sub-mammary fold, corresponding to the inferior border of the pectoralis major muscle and a proportional, harmonious abdominal contouring that consider all related aesthetic units.

At the end of the study the authors may state that in practice male population prefer not to undergo a major operation, such as circumferential abdominoplasty, and be left with significant residual deformity of their breast. A single stage total body lift is best indicated in patients who need abdominoplasty and having gynecomastia correction.

A single stage is an advance in body contouring surgery as patients have the opportunity to benefit from a major body restoration with a single operation. Contouring of the male chest and abdomen by combination of bilateral mastopexy with inferior based dermo glandular pedicle technique and circumferential abdominoplasty in treating pseudogynecomastia post massive weight loss is safe to perform to achieve high patient satisfaction.

\section{References}

1. Ziegler UE, Lorenz U, Daigeler A, et al. (2018) Modified treatment algorithm for pseudogynecomastia after massive weight loss. Ann Plast Surg 81: 290-294.

2. Wiedner M, Justich I, Vasilyeva A, et al. (2013) Complications in body-contouring procedures with special regard to massive weight loss patients: personal observations. European Surgery 45: 75-79.

3. Stevens WG, Repta R, Pacella SJ, et al. (2009) Safe and consistent outcomes of successfully combining breast surgery and abdominoplasty: An Update. Aesthetic Surgery Journal 29: 129-134.

4. Winocour J, Gupta V, Ramirez JR, et al. (2015) Abdominoplasty: Risk factors, complication rates, and safety of combined procedures. Plast Reconstr Surg 136: 597e-606e.

5. Gusenoff JA, Coon D, Rubin JP (2008) Pseudogynecomastia after massive weight loss: Detectability of technique, patient satisfaction, and classification. Plast Reconstr Surg 122: 1301-1311.

6. Al-Rasheed A, Makkar R, Ghazal M (2020) Body Contouring: Full Lower Circumferential Torsoplasty. J Aesthet Reconstr Surg 6: 2.

7. Rohrich RJ, Gosman AA, Conrad MH, et al. (2006) Simplifying circumferential body contouring: The central body lift evolution. Plast Reconstr Surg 118: 525-535.

8. Dindo D, Demartines N, Clavien PA (2004) Classification of surgical complications: a new proposal with evaluation in a cohort of 6336 patients and results of a survey. Ann Surg 240: 205-213.

9. https://www.plasticsurgery.org/documents/News/ Statistics/2018/cosmetic-procedures-men-2018.pdf

10. Michot A, Alet JM, Pélissier P, et al. (2016) Morbidity in combined-procedure associating abdominoplasty and breast surgery: A systematic review. Ann Chir Plast Esthét 61: e9-e19.

11. Grazer FM (1994) Abdominoplasty. In: McCarthy, et al. Plastic Surgery: The trunk and lower extremity. W. B. Saunders Company 6: 3929-3963.

12. Hurwitz DJ (2004) Single-staged total body lift after massive weight loss. Ann Plast Surg 52: 435-441.

13. Rao YV (1969) Augmentation mammaplasty and abdominoplasty in one stage. Plast Reconstr Surg 43: 148-151.

14. Gmür RU, Banic A, Erni D (2003) Is it safe to combine abdominoplasty with other dermolipectomy procedures to correct skin excess after weight loss?. Annals of Plastic Surgery 51: 353-357. 